\title{
BMJ Open CopenFast trial: Faster-acting insulin Fiasp versus insulin NovoRapid in the treatment of women with type 1 or type 2 diabetes during pregnancy and lactation - a randomised controlled trial
}

To cite: Nørgaard SK,

Mathiesen ER, Nørgaard K, et al. CopenFast trial: Faster-acting insulin Fiasp versus insulin NovoRapid in the treatment of women with type 1 or type 2 diabetes during pregnancy and lactation - a randomised controlled trial. BMJ Open 2021;11:e045650. doi:10.1136/ bmjopen-2020-045650

- Prepublication history for this paper is available online. To view these files, please visit the journal online (http://dx.doi org/10.1136/bmjopen-2020045650).

Received 08 0ctober 2020 Revised 01 March 2021 Accepted 22 March 2021
D) Check for updates

(c) Author(s) (or their employer(s)) 2021. Re-use permitted under CC BY-NC. No commercial re-use. See rights and permissions. Published by BMJ.

For numbered affiliations see end of article.

Correspondence to Sidse Kjærhus Nørgaard; sidse.kjaerhus.noergaard.01@ regionh.dk

\section{ABSTRACT}

Introduction Faster-acting insulin aspart (Fiasp) is approved for use in pregnancy and lactation, but no clinical study has evaluated its effects during this life stage in women with pre-existing diabetes. The aim of the CopenFast trial is to evaluate the effect of Fiasp compared with insulin aspart (NovoRapid) on maternal glycaemic control during pregnancy, delivery and lactation and on fetal growth and infant health.

Methods and analysis An open-label randomised controlled trial of pregnant women with type 1 or type 2 diabetes including women on multiple daily injection (MDI) therapy or insulin pump therapy. During a 2-year inclusion period, approximately 220 women will be randomised 1:1 to Fiasp or NovoRapid in early pregnancy and followed until 3 months after delivery. At 9, 21 and 33 gestational weeks and during planned induction of labour or caesarean section, women are offered blinded continuous glucose monitoring (CGM) for 7 days. Randomisation will stratify for type of diabetes and insulin treatment modality (MDI or insulin pump therapy, respectively). Health status of the infants will be followed until 3 months of age. The primary outcome is birth weight SD score adjusted for gestational age and gender. Secondary outcomes include maternal glycaemic control including glycated haemoglobin, preprandial and postprandial self-monitored plasma glucose levels, episodes of mild and severe hypoglycaemia, maternal gestational weight gain and weight retention, CGM time spent in, above and below target ranges as well as pregnancy outcomes including pre-eclampsia, preterm delivery, perinatal mortality and neonatal morbidity. Data analysis will be performed according to the intention-to-treat principle.

Ethics and dissemination The trial has been approved by the Regional Ethics Committee (H-19029966) on 7 August 2019. Results will be sought disseminated in peerreviewed journals and at scientific meetings.

Trial registration number NCT03770767

\section{INTRODUCTION}

In women with type 1 or type 2 diabetes (preexisting) fetal overgrowth is prevalent and up to $50 \%$ of the infants are overweight at
Strengths and limitations of this study

- The CopenFast trial is the first randomised controlled trial that evaluates the effect of Fiasp compared with NovoRapid in women with type 1 or type 2 diabetes during pregnancy, delivery and lactation.

- Both women using multiple daily injection therapy and insulin pump therapy are included in early pregnancy and followed until 3 months after delivery.

- The CopenFast trial is adapted to the routine clinical care setting covering a large and unselected patient population.

- Women are offered blinded continuous glucose monitoring three times in pregnancy and during delivery and the period immediately after delivery.

- Limitations are the single-centre, open-label trial design and lack of registration of diet and physical activity.

birth. ${ }^{12}$ Fetal overgrowth is a common cause of preterm delivery, shoulder dystocia and birth canal lacerations ${ }^{3}$ and high glucose levels and glycated haemoglobin (HbA1c) in the second and third trimesters are well-known predictors. ${ }^{4-8}$ The long-term implications of fetal overgrowth in offspring of women with pre-existing diabetes include future risk of obesity and metabolic syndrome. ${ }^{9}$

Appropriate insulin treatment, parallel to medical nutrition treatment with carbohydrate counting, are cornerstones in obtaining near-normal glucose levels in pregnant and lactating women with pre-existing diabetes. ${ }^{10-12}$ Fetal overgrowth and pregnancy-related complications may be more strongly associated with postprandial plasma glucose excursions compared with fasting plasma glucose levels. ${ }^{13}$ This knowledge together with new pharmacological options have resulted in the attention to 
postprandial glucose excursions as a potential target in the management of diabetes in pregnancy.

The first generation rapid-acting insulin analogues were developed to control postprandial glucose levels by fast onset and short duration of action. A randomised controlled trial (RCT) in pregnant women with type 1 diabetes demonstrated that insulin aspart (NovoRapid) reduced postprandial plasma glucose levels with similar risk of maternal hypoglycaemia compared with soluble human insulin. ${ }^{14} 15$

Faster-acting insulin aspart (Fiasp) is insulin aspart in an innovative formulation in which two excipients have been added, vitamin $\mathrm{B}_{3}$ (niacinamide), to increase the speed of absorption, and a naturally occurring amino acid (L-arginine), for stability. ${ }^{16}$ In non-pregnant adults with type 1 diabetes, Fiasp compared with NovoRapid, resulted in slightly improved HbAlc and significantly reduced postprandial glucose values in multiple daily injection (MDI) treated individuals ${ }^{1718}$ and reduced postprandial glucose values with unchanged HbA1c in insulin pump treated individuals. ${ }^{19}$ In Europe, Fiasp is approved for use in pregnancy and lactation..$^{1620}$

Diabetes management during delivery and lactation in women with type 1 diabetes is scarcely covered in the literature. Use of insulin pump therapy and continuous glucose monitoring (CGM) is safe during delivery and a formal protocol regarding the management of diabetes during delivery is associated with improved intrapartum maternal glucose control. ${ }^{21-23}$ However, there is a gap in our knowledge regarding glycaemic patterns immediately after and during the first weeks after delivery, where the risk of hypoglycaemia is thought to be most pronounced, although this has only been documented in small series of lactating women with type 1 diabetes. ${ }^{24-26}$ In women with type 1 diabetes glucose levels remained over $4.0 \mathrm{mmol} / \mathrm{L}$ after the majority of breast feedings, ${ }^{27} 28$ and in women with normal glucose tolerance suckling did not affect glucose profiles whether the women were fasting or not. ${ }^{29} 30$

To date, no clinical trial has evaluated the effect of Fiasp on glycaemic control and pregnancy outcomes in an unselected cohort of women with pre-existing diabetes during pregnancy and lactation. We hypothesise that Fiasp improves maternal glycaemic control and thereby reduces fetal overgrowth in women with pre-existing diabetes during pregnancy and that Fiasp furthermore improves maternal glycaemic control during delivery and lactation.

The CopenFast trial has been initiated to evaluate the effect of Fiasp compared with NovoRapid on maternal glycaemic control during pregnancy, delivery and lactation and on fetal growth and infant health.

\section{METHODS AND ANALYSIS}

\section{Overall study trial design}

The CopenFast trial is an investigator-initiated, singlecentre, open-label, superiority RCT including pregnant women with pre-existing diabetes during an expected 2-year inclusion period from 11 November 2019. The trial
Box 1 Inclusion and exclusion criteria for participants in the CopenFast trial

\section{Inclusion criteria}

- Women, age $\geq 18$ years.

- Duration of type 1 diabetes (or mature onset of diabetes in the young) $\geq 12$ months.

- Type 2 diabetes (any duration).

- Pregnant with an intrauterine singleton living fetus confirmed by an ultrasound scan between $8+0$ to $13+6$ gestational weeks.

- Routine use of insulin pump therapy, insulin detemir, insulin degludec, insulin glargine, insulin Abasaglar, insulin Toujeo or Neutral Protamine Hagedorn insulin and willing to continue routine treatment modality.

- Women with type 1 diabetes using an insulin pump compatible with trial products.

- Women with type 2 diabetes treated with diet, oral antidiabetic therapy or premixed insulin before pregnancy and willing to change to trial medication according to randomisation or to an appropriate long-acting insulin analogue, as indicated.

- Proficiency in Danish to understand oral and written information.

\section{Exclusion criteria}

- Severe mental or psychiatric barriers or concurrent disease on the decision of the principal investigator.

site is Center for Pregnant Woman with Diabetes, Rigshospitalet, Copenhagen, Denmark. Eligible women are randomised to Fiasp or NovoRapid, either alone (insulin pump therapy) or in combination with usual long-acting insulin (MDI therapy). Each woman participates in the trial from the time of inclusion in early pregnancy to 3 months after delivery. The health of each woman and child will be followed until 3 months after delivery. Inclusion and exclusion criteria are listed in box 1 . The trial protocol has been prepared according to the Standard Protocol Items: Recommendations for Interventional Trials statement. ${ }^{31}$ Approval from the Regional Ethics Committee was obtained on 7 August 2019.

Primary research questions

1. Does treatment with Fiasp result in reduced fetal overgrowth (evaluated by birth weight SD score) when compared with NovoRapid, either alone (insulin pump therapy) or in combination with usual long-acting insulin (MDI therapy)?

2. Is infant growth at 3 months of age more appropriate (evaluated by weight SD score) in offspring of women treated with Fiasp compared with offspring of women treated with NovoRapid?

\section{Screening procedure and recruitment}

All women with pre-existing diabetes are screened and assessed for eligibility at the first antenatal visit at Center for Pregnant Women with Diabetes, where eligibility assessment is conducted by an investigator. Women who meet the eligibility criteria (box 1) are carefully informed of the trial and asked if interested in participation. If interested, women are given both oral and written participant information about the trial and time to consider 


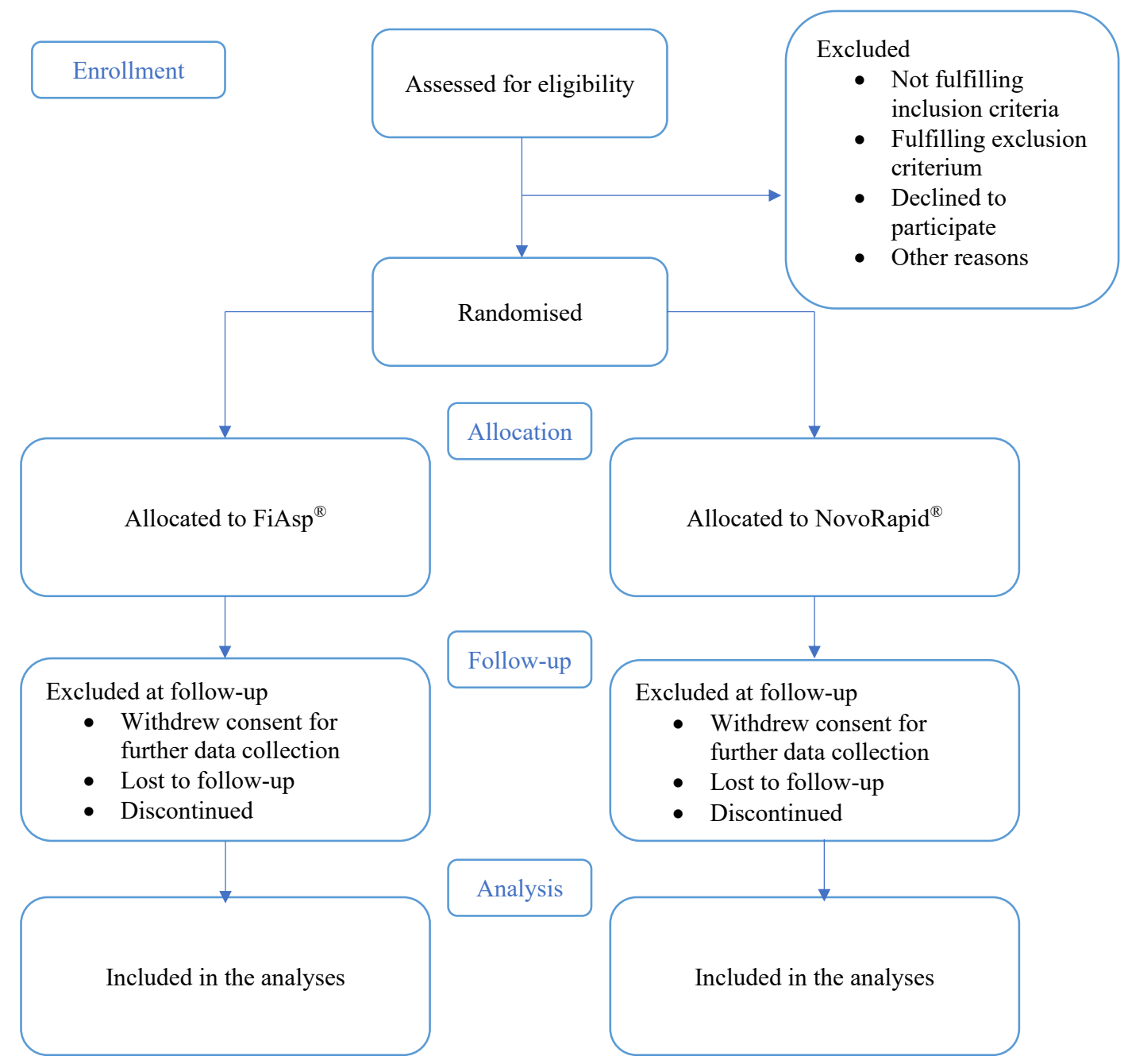

Figure 1 Flow chart of the CopenFast trial design.

participation. Women who participate are asked to provide written informed consent prior to commencing trial participation. Written consent from their partner regarding data collection of the infant, is also obtained. Informed consent is obtained by an investigator. Inclusion and exclusion of participants will be reported according to the Consolidated Standards of Reporting Trials (CONSORT) statement ${ }^{32}$ (figure 1).

Women attending the centre in very early pregnancy, before $8+0$ weeks (week+days), are informed as above and randomised when eligible at $\geq 8+0$ weeks.

\section{Randomisation}

Women are randomised from $8+0$ to $13+6$ weeks. Randomisation takes place no later than 21 days after the screening visit at which the woman was confirmed eligible and consented to participate provided informed consent. Women are randomised according to a computergenerated randomisation sequence in permuted blocks of varying sizes $(2,4$ or 6$)$ stratified by diabetes type and treatment modality (type 1 diabetes vs type 2 diabetes and MDI therapy vs insulin pump therapy, respectively).

The randomisation sequence has been prepared and is kept by staff from the hospital pharmacy of the Capital Region. Allocation concealment is ensured by sequentially numbered, opaque and sealed envelopes.

Personal information about potential participants will be kept to a minimum and kept in a secured screening log. Each participant will be allocated a unique three-digit participation ID number and randomisation number.

\section{Intervention}

All women routinely consult a diabetes specialist approximately every 2 weeks throughout pregnancy. If a woman at randomisation is to change to another type of rapidacting insulin analogue, she is given the same number of insulin units as was intended with the former insulin type. All women are recommended medical nutrition treatment with carbohydrate counting and to inject the trial product immediately before each meal, regardless 
of insulin type. ${ }^{10}{ }^{12}$ In case of large postprandial glucose excursions or suspected delayed insulin absorption, especially in late pregnancy, the trial product can be injected up to $30 \mathrm{~min}$ before meals, regardless of insulin type. The dose of trial product is titrated based on self-monitored plasma glucose (SMPG) and/or CGM glucose values when applicable, at the clinical visits throughout the study and women are encouraged to adjust trial product dose every 3-5 days when appropriate between clinical visits at our centre, as part of routine care.

The recommendations on medical nutritional therapy and physical activity are the same, regardless of randomisation. The women are recommended carbohydrate counting and regular meals to match the amount of trial product. A daily carbohydrate intake of $150 \mathrm{~g}$ from the main sources (bread, whole grain, dairy products, fruits, rice, potatoes and pasta) and $25 \mathrm{~g}$ from other carbohydrate sources (ie, vegetables) is recommended, distributed as $20 \mathrm{~g}, 40-50 \mathrm{~g}$ and $40-50 \mathrm{~g}$ for breakfast, lunch and dinner, respectively, and $10 \mathrm{~g}$ from two to four snacks. Physical activity for at least $30 \mathrm{~min}$ per day is recommended. ${ }^{1012}$

At each trial visit adherence to the intervention will be monitored. Non-adherence to trial products will be noted as a trial protocol deviation and reported to the principal investigator. To improve adherence participants will be sought provided with the appropriate amount of trial product and participants are informed about the possibility to be provided with trial products between trial visits, when attending the Center for Pregnant Women with Diabetes.

The newborns are routinely roomed-in with their mothers. ${ }^{33}$ The newborns receive assistance in the neonatal intensive care unit only in case of neonatal morbidity (severe neonatal hypoglycaemia, severe jaundice or respiratory distress) and/or symptoms of prematurity.

\section{Data collection}

\section{Inclusion}

Baseline data are recorded: Age, ethnicity, smoking status, self-reported prepregnancy weight, actual weight, height, parity, gestational age, duration of diabetes, urinary ketones, urinary albumin excretion, other medication and folic acid intake, prepregnancy HbA1c, actual HbA1c, blood pressure, insulin treatment modality, insulin dose and insulin pump settings before pregnancy and at inclusion, hypoglycaemia awareness status, number of mild hypoglycaemic events (symptoms familiar to the woman as hypoglycaemia and managed by herself) ${ }^{34} 35$ during the previous week and severe hypoglycaemic events (requiring assistance from others to restore normal glucose levels) ${ }^{36}$ in the year preceding pregnancy and since confirmation of pregnancy. Retinal photo screening performed at inclusion and according to routine practice. ${ }^{37}$

\section{Trial visits at 21,33 and 36 weeks}

Gestational age, weight, HbAlc, blood pressure, insulin dose, insulin pump settings, urinary ketones, urinary albumin excretion and number of mild and severe hypoglycaemic events will be noted.

\section{At delivery}

Date of delivery, miscarriage, fetal loss (after $22+0$ weeks), early preterm delivery (before $34+0$ weeks), preterm delivery (before $37+0$ weeks), pre-eclampsia and mode of delivery (vaginal, instrumental, emergency and planned caesarean section) will be noted. The following will be registered regarding the infant: gender, gestational age at delivery, head circumference, abdominal circumference, weight and length at birth. Neonatal morbidity (severe neonatal hypoglycaemia, severe jaundice, respiratory distress and duration of stay in neonatal intensive care unit) and neonatal mortality will also be registered.

\section{Trial visits 1 and 3 months after delivery}

Weight, HbA1c, insulin dose, insulin pump settings, number of mild hypoglycaemic events in the previous week and number of severe hypoglycaemic events since delivery will be noted. The following will be noted regarding the infants: Length, weight, presence of congenital malformations and days with hospitalisation since discharge after delivery.

\section{Other data collection}

Concomitantly prescribed medication (including trade name or generic name, indication, dose, start date and stop date or continuation), intercurrent illness and adverse events are recorded at inclusion and at each trial visit.

\section{Questionnaires}

Questionnaires are completed at inclusion, at 33 weeks as well as 1 and 3 months after delivery where age, smoking status, years of education, self-estimated hypoglycaemia awareness status, number of events of mild hypoglycaemia the previous week and number of events of severe hypoglycaemia since last completed questionnaire as well as lactation status are reported when indicated. If a woman experiences severe hypoglycaemia a structured questionnaire about the event is filled in as soon after the event as possible. $^{2734}$

\section{Data on diabetes technology}

For women using insulin pump therapy or a bolus calculator system the type will be noted. Basal insulin delivery rates, insulin-carbohydrate ratio and insulin sensitivity factor with time intervals, insulin duration time and target glucose are recorded at each trial visit. ${ }^{38}{ }^{39}$ Insulin duration time is typically set to 4 hours. Total daily insulin dose including proportion of basal and bolus doses, and total daily carbohydrate intake entered in the insulin pump are also recorded. Women on insulin pump therapy, compatible with trial products, continue with the same type of pump as prior to pregnancy, regardless of type. We expect that few women will use sensor augmented pumps, hybrid closed loop systems or do-it-yourself closed loop technology. ${ }^{40} 41$ In general, women are recommended not 
to use suspension before low, however suspend on low can often be maintained at a threshold of $4.0 \mathrm{mmol} / \mathrm{L}$.

All women are offered blinded CGM (Envision Pro Sensor, Medtronic MiniMed, Northridge, CA 91325, USA) at inclusion, at the trial visits at approximately 21 and 33 weeks and in the morning for planned induction of labour or caesarean section. Blinded CGM is offered for 7 days, the measurement period provided by the Envision Pro Sensor. The CGM will be applied when attending Center for Pregnant Women with Diabetes.

Women who do not wish to wear a blinded CGM device, and already use an open CGM routinely, continue with the same type of CGM during pregnancy, delivery and lactation. The CGM data on women who routinely use open CGM will be collected for the same 7 days periods as the blinded CGM for trial purpose. The type of CGM device used will be noted.

\section{Self-monitoring of plasma glucose}

All women, including those using CGM routinely, are asked to routinely perform SMPG seven times daily: Immediately before each main meal, 11/2 hours after initiating a main meal and before bedtime. Insulin dosing is adjusted weekly throughout pregnancy aiming for target SMPG $4.0-5.5 \mathrm{mmol} / \mathrm{L}$ preprandially and $4.0-7.0 \mathrm{mmol} / \mathrm{L}$ postprandially during pregnancy. During delivery, all women are recommended glucose target of $4.0-7.0 \mathrm{mmol} / \mathrm{L}$ according to local protocol and regardless of mode of delivery. ${ }^{42}$ During lactation the SMPG targets are $4.0-7.0 \mathrm{mmol} / \mathrm{L}$ preprandially and $6.0-10.0 \mathrm{mmol} / \mathrm{L}$ before bedtime. At inclusion, at 21 and 33 weeks, women are encouraged to perform 7 days SMPG profiles for trial purpose. Number and percentages of glucose measurements $<3.0 \mathrm{mmol} / \mathrm{L}$ in each SMPG profile is recorded.

\section{Outcomes}

The primary outcome is offspring birth weight SD score adjusted for gestational age and gender. ${ }^{43}$

\section{Secondary outcomes}

Maternal outcomes

- HbAlc levels at each trial visit during pregnancy and at 1 and 3 months after delivery.

- Postprandial and preprandial SMPG levels during pregnancy.

- The percentage of glucose readings during blinded CGM and/or any routine open CGM use in the target range $3.5-7.8 \mathrm{mmol} / \mathrm{L},<3.5 \mathrm{mmol} / \mathrm{L}$ and $>7.8 \mathrm{mmol} / \mathrm{L}$ at night-time $(11.00 \mathrm{pm}$ up to and including $06.59 \mathrm{am}$ ) and over 24 hours, respectively, at each trial visit during pregnancy and around delivery. ${ }^{44}$

- The percentage of glucose readings during blinded CGM and/or any routine open CGM use in the first 1 week period after delivery in the target range $3.9-10.0 \mathrm{mmol} / \mathrm{L},<3.9 \mathrm{mmol} / \mathrm{L}$ and $>10.0 \mathrm{mmol} / \mathrm{L}$ at night-time (11.00 pm up to and including $06.59 \mathrm{am}$ ) and over 24 hours, respectively. ${ }^{44}$
- The number of episodes with mild hypoglycaemia in the previous week in early and late pregnancy and at 1 and 3 months after delivery.

- The prevalence of severe hypoglycaemia in the year preceding pregnancy, during pregnancy and the first 3 months after delivery.

- Maternal weight gain during pregnancy defined as the difference between the last weight measured before delivery and the self-reported prepregnancy weight $(\mathrm{kg}){ }^{45}$

- Maternal gestational weight gain retention 3 months after delivery defined as $>5.0 \mathrm{~kg}$ compared with prepregnancy weight. ${ }^{46}$

- Insulin pump settings during pregnancy, around delivery and during lactation.

- Insulin treatment during pregnancy, around delivery and during lactation.

Pregnancy outcomes

- The prevalence of miscarriage.

- Mode of delivery (vaginal, emergency or planned caesarean section, instrumental delivery).

- Early preterm delivery (before $34+0$ weeks) and preterm delivery (before $37+0$ weeks).

- Pre-eclampsia.

- Perinatal death (from 22+0 weeks to 7 days after birth).

Infant outcomes

- The prevalence of fetal overgrowth, defined as the offspring birth weight SD score +1.28 equivalent to large for gestational age. ${ }^{43}$

- Infant growth evaluated by weight SD score at 1 and 3 months of age.

- Neonatal morbidity (severe neonatal hypoglycaemia, severe jaundice or respiratory distress).

- Admission to and duration of stay in neonatal intensive care unit in case of neonatal morbidity and/or symptoms of prematurity.

- Infant morbidity evaluated as hospitalisation during the first 3 months of life (after discharge in the neonatal period).

\section{Statistical analysis}

Baseline data will be presented stratified by treatment allocation. Categorical variables will be presented as numbers (\%) and numerical variables will be presented as medians (IQRs) or means (SD) as appropriate.

The primary data analyses will include all randomised women and be conducted according to the intentionto-treat principle. The primary outcome, birth weight SD score in the two treatment groups and numerical outcomes will be analysed by multiple linear regression adjusted for the stratification variable used in randomisation and presented as mean differences with 95\% CIs and $\mathrm{p}$ values. If the assumptions for linear regression are not met, data will be analysed with a corresponding non-parametric test adjusted for the stratification variable. Categorical outcomes will be analysed using logistic regression analyses adjusted for 
the stratification variable and presented as ORs with 95\% CIs and $\mathrm{p}$ values.

Only women who withdrew consent for further data collection will be excluded. The amount of missing data will be presented for each variable and depending on the amount of missing data all analyses will be conducted accordingly, that is, as complete-case analyses excluding patients with missing data for one or more used variables in said analysis if reasonable. Per-protocol analyses will be conducted as indicated.

Additional separate subgroup analyses of women with type 1 diabetes on MDI or insulin pump therapy and women with type 2 diabetes are planned. Within the group of women with type 1 diabetes a separate exploratory analysis of women on MDI therapy and insulin pump therapy, respectively, will be performed. Additional exploratory analyses will be performed on the data set as appropriate and it will be emphasised which analyses are made post hoc.

Two-sided $p$ values $<0.05$ will be regarded as statistically significant.

Results will be reported in accordance with the CONSORT statement regardless of findings. ${ }^{32}$

\section{Sample size estimation}

Based on the assumption that, in our population, the mean offspring birth weight SD score is +0.8 (SD 1.6) ${ }^{47}$ and that use of Fiasp can reduce the mean offspring birth weight SD score to +0.2 , and a type 1 error of $5 \%$ and a type 2 error of $20 \%$, the number of patients needed in each arm is 99 . On an annual basis, approximately 150 women with pre-existing diabetes (90 with type 1 diabetes and 60 with type 2 diabetes) are followed at Center for Pregnant Women with Diabetes during pregnancy. The 2-year inclusion period is planned to secure a sufficient number of women with pregnancies beyond 22 weeks. We expect to screen a total of 300 women with type 1 or type 2 diabetes and to randomise a total of approximately 220 women.

\section{Monitoring and safety considerations}

An independent safety assessment and monitoring plan in accordance with the Good Clinical Practice (GCP) guidelines is followed including relevant reporting to Danish Medicines Agency and ethics committee. Safety of participants and trial products is continually and at each trial visit evaluated. If side effects and/or adverse events are reported, date and type are noted in the patient record and events are reported according to local regulations.

\section{Precautions during the COVID-19 pandemic}

Based on guidelines from the local authorities, inclusion was paused from 12 March 2020 until 1 May 2020 and CGM in included women was not applied to minimise direct physical contact between the caregiver and patient. All other data collection continued as they were part of routine care or telephone consultation and in accordance with national and local guidelines including limitation of close physical contact, proper distance and frequent sanitising and disinfection.
Availability of data and material

All original data will be handled and secured by the research group for 15 years after the trial has ended, according to The Danish Data Protection Agency. The CopenFast trial has a data sharing policy and reasonable requests will be handled as appropriate.

\section{Public and patient involvement}

Women with pre-existing diabetes were not involved in planning of this study protocol.

\section{ETHICS AND DISSEMINATION}

Approval from the Regional Ethics Committee (Protocol number H-19029966, approval date 7 August 2019), The Danish Medicines Agency (Protocol number 2018004680-31, approval date 20 May 2019) and The Danish Data Protection Agency (Protocol number VD-2019-244, approval date 23 May 2019) have been obtained. The trial is performed in accordance with the principles in the Helsinki Declaration II and International Conference on Harmonisation Good Clinical Practice. The trial is conducted according to the protocol and is monitored by the GCP unit at University of Copenhagen independently from the investigators. All aspects of the trial may be subject to inspection from the Danish Medicines Agency and the regional ethics committee. Audits and inspections may take place during or after the trial. Important protocol modifications will be reported to The National Committee on Health Research Ethics, The Danish Medicines Agency and the local GCP unit. Data are entered in a REDCap (Research Electronic Data Capture) database by SKN and LR. The database can only be accessed with a double code. To promote data quality range checks for data values are set and double data entry is performed in a random sample of participants. Data will be posted on public websites including ClinicalTrials.gov where required to comply with regulations. Patients are covered by the public patient insurance at Rigshospitalet and will be covered by product liability insurance for the trial products. Each participant in the trial signed a written consent form prior to participation.

The CopenFast trial will be the first RCT to evaluate the effect of Fiasp compared with NovoRapid in an unselected cohort of women with pre-existing diabetes during pregnancy, delivery and lactation and in their offspring.

With a study design adapted to routine clinical care and with broad inclusion criteria and few exclusion criteria we expect to include the majority of a population of unselected women from a large, geographically well-defined region. The trial setting and data collection is integrated in the daily routine clinical care provided in Center for Pregnant Women with Diabetes. A possible limitation of the study is the single-centre design however, the generalisability may be potentially large due to the mainly unselected patient population. Another limitation may be the open-label design, which may increase the risk of bias. ${ }^{48}$ However, the allocation sequence concealment and an 
objective primary outcome may secure that this trial is only modestly affected by bias. Finally, the lack of diet and physical activity registration might also be a limitation. However, our experience is that assessment of dietary intake and documentation of physical activity is difficult to obtain and less precise, even in a research setting with pregnant women with pre-existing diabetes and may limit the recruitment of an unselected cohort. This may lead to bias by mainly obtaining data from the most resourceful women. ${ }^{27} 49$

The trial includes women with pre-existing diabetes and covers both MDI and insulin pump therapy. This will allow exploratory comparisons between various insulin treatment modalities in pregnancy and during lactation up to 3 months after delivery. Thereby it may be possible to explore whether Fiasp will be more helpful in certain subgroups of participants, that is, insulin pump users. Women are offered blinded CGM three times in pregnancy and around delivery to evaluate the potential of CGM on glycaemic control and fetal overgrowth as well as glycaemic patterns during and immediately after delivery. The blinded CGM provides data for a 7-day period; however, insulin therapy during pregnancy is dynamic and rapidly changing. In our centre, adjustment of insulin therapy takes place at each clinical visit every 2 weeks and women are routinely advised to adjust insulin doses every $3-5$ days between clinical visits.

The results of the trial are expected to be disseminated in peer-reviewed journals and at national and international scientific meetings regardless of findings. All participants who have consented to receiving the results of the trial will receive a summary of the findings expressed in lay terms. No professional writers will be used.

In summary, in this RCT on a mainly unselected cohort of women with pre-existing diabetes during pregnancy, delivery and lactation the primary outcome is birth weight SD score.

The trial will also examine other outcomes including maternal glycaemic control, insulin dose and the most appropriate insulin pump settings during pregnancy, around and immediately after delivery and during lactation as well as important pregnancy outcomes including pre-eclampsia, preterm delivery and neonatal morbidity. The potential of CGM data to predict fetal growth will be evaluated. The investigators expect that the trial will provide valuable new information on relevant maternal, pregnancy and infant-related endpoints to inform future prescribing with Fiasp and NovoRapid during pregnancy, around and immediately after delivery and during lactation in women with pre-existing diabetes.

\footnotetext{
Author affiliations

${ }^{1}$ Center for Pregnant Women with Diabetes, Rigshospitalet, Copenhagen, Denmark

${ }^{2}$ Department of Endocrinology, Rigshospitalet, Copenhagen, Denmark

${ }^{3}$ Department of Clinical Medicine, University of Copenhagen Faculty of Health and

Medical Sciences, Copenhagen, Denmark

${ }^{4}$ Steno Diabetes Center Copenhagen, Gentofte, Denmark

${ }^{5}$ Department of Gynaecology and Obstetrics, Nordsjaellands Hospital, Hillerod,

Denmark

${ }^{6}$ Department of Obstetrics, Rigshospitalet, Copenhagen, Denmark
}

Acknowledgements The authors would like to thank Ann-Sofi Lunde, Birgitta Ellingsgaard, Charlotte Barfred, Helle Løvschall, Signe Lou-Møller and Vibeke Ladefoged Nichum, registered nurses at Center for Pregnant Women with Diabetes at Rigshospitalet for their valuable support, effort and involvement in the trial. The CopenFast trial is sponsored by Novo Nordisk. Trial products are purchased at the cost negotiated with Hospital Pharmacy of the Capital Region, Denmark. The Envision Pro Sensor devices are purchased at the cost negotiated with Medtronic.

Contributors SKN and LR wrote the first draft of the manuscript and have access to the final trial set. The trial was initiated by the principal investigator LR in collaboration with senior investigators ERM and PD and coordinating investigator SKN. SKN, ERM, KN, TDC, PD and LR all contributed to the concept of the trial. ERM, KN, TDC and PD critically read and revised the manuscript. All authors have approved the manuscript and consented to publication of the manuscript prior to submission.

Funding The trial is funded by a Novo Nordisk grant covering direct costs of the trial including trial products as well as salary for a PhD student (SKN) as well as salaries during a $3 \frac{1}{2} 2$-year trial period for a trial nurse and some part-time salary for principal investigator LR and investigator ERM. Novo Nordisk has not been involved in initiation of the trial or the study design and has no involvement in terms of collection, analysis and interpretation of the data and the decision to submit future results for publication. The funding will be paid to a research account at Department of Endocrinology PE7562, Rigshospitalet. Grant number: U1111-1209-6358.

Competing interests None declared.

Patient and public involvement Patients and/or the public were not involved in the design, or conduct, or reporting, or dissemination plans of this research.

Patient consent for publication Not required.

Provenance and peer review Not commissioned; externally peer reviewed.

Open access This is an open access article distributed in accordance with the Creative Commons Attribution Non Commercial (CC BY-NC 4.0) license, which permits others to distribute, remix, adapt, build upon this work non-commercially, and license their derivative works on different terms, provided the original work is properly cited, appropriate credit is given, any changes made indicated, and the use is non-commercial. See: http://creativecommons.org/licenses/by-nc/4.0/.

\section{ORCID iD}

Sidse Kjærhus Nørgaard http://orcid.org/0000-0002-6664-5755

\section{REFERENCES}

1 Secher AL, Parellada CB, Ringholm L, et al. Higher gestational weight gain is associated with increasing offspring birth weight independent of maternal glycemic control in women with type 1 diabetes. Diabetes Care 2014;37:2677-84.

2 Murphy HR, Bell R, Cartwright C, et al. Improved pregnancy outcomes in women with type 1 and type 2 diabetes but substantial clinic-to-clinic variations: a prospective nationwide study. Diabetologia 2017;60:1668-77.

3 Weissmann-Brenner A, Simchen MJ, Zilberberg E, et al. Maternal and neonatal outcomes of large for gestational age pregnancies. Acta Obstet Gynecol Scand 2012;91:844-9.

4 Glinianaia SV, Tennant PWG, Bilous RW, et al. $\mathrm{HbA}(1 \mathrm{c})$ and birthweight in women with pre-conception type 1 and type 2 diabetes: a population-based cohort study. Diabetologia 2012;55:3193-203.

5 Maresh MJA, Holmes VA, Patterson CC, et al. Glycemic targets in the second and third trimester of pregnancy for women with type 1 diabetes. Diabetes Care 2015;38:34-42.

6 Persson M, Pasupathy D, Hanson U, et al. Birth size distribution in 3,705 infants born to mothers with type 1 diabetes: a populationbased study. Diabetes Care 2011;34:1145-9.

7 Jensen D, Damm P, Moelsted-Pedersen L, et al. Outcomes in type 1 diabetic pregnancies. Diabetes Care 2004;27:2819-23.

8 Clausen TD, Mathiesen E, Ekbom P, et al. Poor pregnancy outcome in women with type 2 diabetes. Diabetes Care 2005;28:323-8.

9 Clausen TD, Mathiesen ER, Hansen T, et al. Overweight and the metabolic syndrome in adult offspring of women with diet-treated gestational diabetes mellitus or type 1 diabetes. J Clin Endocrinol Metab 2009;94:2464-70.

10 Ringholm L, Damm P, Mathiesen ER. Improving pregnancy outcomes in women with diabetes mellitus: modern management. Nat Rev Endocrinol 2019;15:406-16. 
11 Ringholm L, Stougaard EB, Nørgaard SK, et al. Diabetes management during breastfeeding in women with type 1 diabetes. Curr Diab Rep 2020;20:34.

12 Roskjær AB, Andersen JR, Ronneby H, et al. Dietary advices on carbohydrate intake for pregnant women with type 1 diabetes. J Matern Fetal Neonatal Med 2015;28:229-33.

13 Jovanovic LG. Using meal-based self-monitoring of blood glucose as a tool to improve outcomes in pregnancy complicated by diabetes. Endocr Pract 2008;14:239-47.

14 Mathiesen ER, Kinsley B, Amiel SA, et al. Maternal glycemic control and hypoglycemia in type 1 diabetic pregnancy: a randomized trial of insulin aspart versus human insulin in 322 pregnant women. Diabetes Care 2007;30:771-6.

15 Hod M, Damm P, Kaaja R, et al. Fetal and perinatal outcomes in type 1 diabetes pregnancy: a randomized study comparing insulin aspart with human insulin in 322 subjects. Am J Obstet Gynecol 2008;198:186.e1-7.

16 Heise T, Stender-Petersen K, Hövelmann U, et al. Pharmacokinetic and pharmacodynamic properties of Faster-Acting insulin aspart versus insulin aspart across a clinically relevant dose range in subjects with type 1 diabetes mellitus. Clin Pharmacokinet 2017;56:649-60.

17 Russell-Jones D, Bode BW, De Block C, et al. Fast-acting insulin aspart improves glycemic control in basal-bolus treatment for type 1 diabetes: results of a 26 -week multicenter, active-controlled, treatto-target, randomized, parallel-group trial (onset 1). Diabetes Care 2017;40:943-50

18 Buse JB, Carlson AL, Komatsu M, et al. Fast-acting insulin aspart versus insulin aspart in the setting of insulin degludec-treated type 1 diabetes: efficacy and safety from a randomized double-blind trial. Diabetes Obes Metab 2018;20:2885-93.

19 Klonoff DC, Evans ML, Lane W, et al. A randomized, multicentre trial evaluating the efficacy and safety of fast-acting insulin aspart in continuous subcutaneous insulin infusion in adults with type 1 diabetes (onset 5). Diabetes Obes Metab 2019;21:961-7.

20 European Medicines Agency. Fiasp summary of product characteristics [online], 2017. Available: https://www.ema.europa.eu/ en/medicines/human/EPAR/fiasp [Accessed 31 Jul 2020].

21 Fresa R, Visalli N, Di Blasi V, et al. Experiences of continuous subcutaneous insulin infusion in pregnant women with type 1 diabetes during delivery from four Italian centers: a retrospective observational study. Diabetes Technol Ther 2013;15:328-34.

22 Drever E, Tomlinson G, Bai AD, et al. Insulin pump use compared with intravenous insulin during labour and delivery: the inspired observational cohort study. Diabet Med 2016;33:1253-9.

23 Dude AM, Niznik C, Peaceman AM, et al. Evaluation of an intrapartum insulin regimen for women with diabetes. Obstet Gynecol 2020;136:411-6.

24 Achong N, Duncan EL, Mclntyre HD, et al. Peripartum management of glycemia in women with type 1 diabetes. Diabetes Care 2014;37:364-71.

25 Inkster B, Elder J, Alexander C, et al. Post-partum hypoglycaemia in lactating women with type 1 diabetes: a pilot study using continuous glucose monitoring. Br J Diabetes 2015;15:119-22.

26 Riviello C, Mello G, Jovanovic LG. Breastfeeding and the basal insulin requirement in type 1 diabetic women. Endocr Pract 2009;15:187-93.

27 Ringholm L, Roskjær AB, Engberg S, et al. Breastfeeding at night is rarely followed by hypoglycaemia in women with type 1 diabetes using carbohydrate counting and flexible insulin therapy. Diabetologia 2019;62:387-98.

28 Achong N, Mclntyre HD, Callaway L, et al. Glycaemic behaviour during breastfeeding in women with type 1 diabetes. Diabet Med 2016;33:947-55.

29 Colatrella A, Framarino M, Toscano V, et al. Continuous glucose monitoring during breastfeeding in women with recent gestational diabetes mellitus. Diabetes Technol Ther 2012;14:576-82.
30 Bentley-Lewis R, Goldfine AB, Green DE, et al. Lactation after normal pregnancy is not associated with blood glucose fluctuations. Diabetes Care 2007;30:2792-3.

31 Chan A-W, Tetzlaff JM, Altman DG, et al. SPIRIT 2013 statement: defining standard protocol items for clinical trials. Ann Intern Med 2013;158:200-7.

32 Schulz KF, Altman DG, Moher D. CONSORT 2010 statement. Obstet Gynecol 2010;115:1063-70.

33 Stage E, Mathiesen ER, Emmersen PB, et al. Diabetic mothers and their newborn infants - rooming-in and neonatal morbidity. Acta Paediatr 2010;99:997-9.

34 Nielsen LR, Pedersen-Bjergaard U, Thorsteinsson B, et al. Hypoglycemia in pregnant women with type 1 diabetes: predictors and role of metabolic control. Diabetes Care 2008;31:9-14.

35 Pedersen-Bjergaard U, Pramming S, Thorsteinsson B. Recall of severe hypoglycaemia and self-estimated state of awareness in type 1 diabetes. Diabetes Metab Res Rev 2003;19:232-40.

36 Seaquist ER, Anderson J, Childs B, et al. Hypoglycemia and diabetes: a report of a workgroup of the American diabetes association and the endocrine society. Diabetes Care 2013;36:1384-95.

37 Vestgaard M, Ringholm L, Laugesen CS, et al. Pregnancy-induced sight-threatening diabetic retinopathy in women with type 1 diabetes. Diabet Med 2010;27:431-5.

38 Bongiovanni M, Fresa R, Visalli N, et al. A study of the carbohydrateto-insulin ratio in pregnant women with type 1 diabetes on pump treatment. Diabetes Technol Ther 2016;18:360-5.

39 Mathiesen JM, Secher AL, Ringholm L, et al. Changes in basal rates and bolus calculator settings in insulin pumps during pregnancy in women with type 1 diabetes. J Matern Fetal Neonatal Med 2014;27:724-8.

40 Lemieux P, Yamamoto JM, Donovan LE. Do-it-yourself artificial pancreas system use in pregnant women with type 1 diabetes in a real-world setting: 2 case reports. Can J Diabetes 2021 doi:10.1016/j. jcjd.2021.01.006

41 Schütz-Fuhrmann I, Schütz AK, Eichner M, et al. Two subsequent pregnancies in a woman with type 1 diabetes: artificial pancreas was a Gamechanger. J Diabetes Sci Technol 2020;14:972-3.

42 Cordua S, Secher AL, Ringholm L, et al. Real-time continuous glucose monitoring during labour and delivery in women with Type 1 diabetes - observations from a randomized controlled trial. Diabet Med 2013:30:1374-81.

43 Marsál K, Persson PH, Larsen T, et al. Intrauterine growth curves based on ultrasonically estimated foetal weights. Acta Paediatr 1996;85:843-8.

44 Battelino T, Danne T, Bergenstal RM, et al. Clinical targets for continuous glucose monitoring data interpretation: recommendations from the International consensus on time in range. Diabetes Care 2019;42:1593-603.

45 Jensen DM, Damm P, Sørensen B, et al. Pregnancy outcome and prepregnancy body mass index in 2459 glucose-tolerant Danish women. Am J Obstet Gynecol 2003;189:239-44.

46 Vinter CA, Jensen DM, Ovesen P, et al. Postpartum weight retention and breastfeeding among obese women from the randomized controlled lifestyle in pregnancy (LiP) trial. Acta Obstet Gynecol Scand 2014:93:794-801.

47 Nørgaard SK, Vestgaard MJ, Jørgensen IL, et al. Diastolic blood pressure is a potentially modifiable risk factor for preeclampsia in women with pre-existing diabetes. Diabetes Res Clin Pract 2018;138:229-37.

48 Moustgaard $\mathrm{H}$, Clayton GL, Jones HE, et al. Impact of blinding on estimated treatment effects in randomised clinical trials: metaepidemiological study. BMJ 2020;368:16802.

49 Ásbjörnsdóttir B, Ronneby $\mathrm{H}$, Vestgaard $\mathrm{M}$, et al. Lower daily carbohydrate consumption than recommended by the Institute of medicine is common among women with type 2 diabetes in early pregnancy in Denmark. Diabetes Res Clin Pract 2019;152:88-95. 